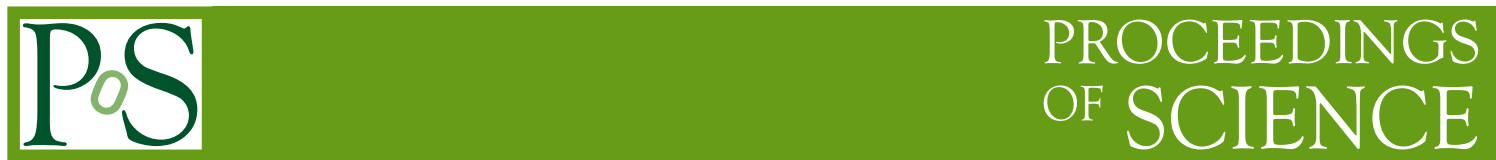

\title{
SOUSA's Swift Supernova Siblings
}

\section{Peter J. Brown*}

George P. and Cynthia Woods Mitchell Institute for Fundamental Physics \& Astronomy, Texas A. \& M. University, Department of Physics and Astronomy, 4242 TAMU, College Station, TX

77843, USA E-mail: pbrown@physics . tamu . edu

Swift has observed over three hundred supernovae in its first ten years. Photometry from the Ultra-Violet Optical Telescope (UVOT) is being compiled in the Swift Optical/Ultraviolet Supernovae Archive (SOUSA). The diversity of supernovae leads to a wide dynamic range of intrinsic properties. The intrinsic UV brightness of supernovae as a function of type and epoch allows one to understand the distance ranges at which Swift can reliably detect supernovae. The large Swift sample also includes supernovae from the same galaxy as other Swift supernovae. Through the first ten years, these families include 34 supernovae from 16 host galaxies (two galaxies have each hosted three Swift supernovae).

Swift: 10 Years of Discovery,

2-5 December 2014

La Sapienza University, Rome, Italy

\footnotetext{
* Speaker.
} 


\section{Swift Supernova Observations}

In the first ten years of Swift operations [1], Swift has observed over three hundred supernovae. All young supernovae (i.e. excluding supernova remnants and others which exploded before the launch of Swift) known by the author to have been observed by Swift are listed on the Swift Supernova website ${ }^{1}$. The observed supernovae cover a wide variety of explosion and environment characteristics. The UV data from the Ultra-Violet/Optical Telescope (UVOT; 2) are being uniformly reduced and publicly released as the Swift Optical/Ultraviolet Supernova Archive (SOUSA). The details of the photometric reduction are available in the cited paper [3].

As we look ahead to the next years of Swift supernova observations, results from the first ten years can guide the choice of future targets and observing strategies. Most of the Swift supernova observations in the first ten years have focused on nearby supernovae with redshifts $\mathrm{z}<0.02$. Figure 1 shows the wide variety of absolute magnitudes in the mid-ultraviolet uvm 2 filter. Distance limits are estimated based on a limiting magnitude of 20 in the UVOT Vega system [4, 5]. Fainter supernovae such as type $\mathrm{Ib} / \mathrm{c}$ and subluminous type Ia supernovae can only be well observed within a redshift of $\mathrm{z} \sim 0.005$. UV-bright type IIP and Ibn supernovae can be observed to $\mathrm{z} \sim 0.05$. Some type Ia supernovae are also this bright, including so-called "Super-Chandrasekhar" type Ia supernovae [6] and the peculiar SN 2011de [7]. Very UV-bright type IIn can be observed to $\mathrm{z} \sim 0.2$ and the type II superluminous supernovae to $\mathrm{z} \sim 0.5$. Expanding the redshift range will increase the volume and numbers of some supernova types which can be observed with Swift. In particular measuring the peak magnitudes of type Ia supernovae in the Hubble flow will reduce the uncertainties in the distances and absolute magnitudes, a problem with some of the nearby Swift supernova samples [8].

\section{Swift Supernova Siblings}

As the Swift supernova sample grows, so does the chance that some of the host galaxies have or will host another supernova. We call these pairings of supernovae from the same hosts "siblings." We reserve the term "twins" for supernovae which are nearly identical in many properties. Supernova twins and siblings are more useful than just the sum of their individual utility.

Supernova twins are useful because their similar spectroscopic properties allow color differences to be traced to line of sight reddening, and their extinction corrected magnitudes can be used to infer distance differences $[9,10]$. SNe 2011by and 2011fe were nearly identical in the optical and the UV differences were attributed to metallicity [11]. Other effects such as density gradients also cause large UV differences while leaving the optical virtually unchanged [12]. Since "twinness" can be very subjective, below we just focus on siblings, since host identification for nearby supernovae is a much more straightforward process.

\footnotetext{
${ }^{1}$ http://swift.gsfc.nasa.gov/docs/swift/sne/swift_sn.html.
} 


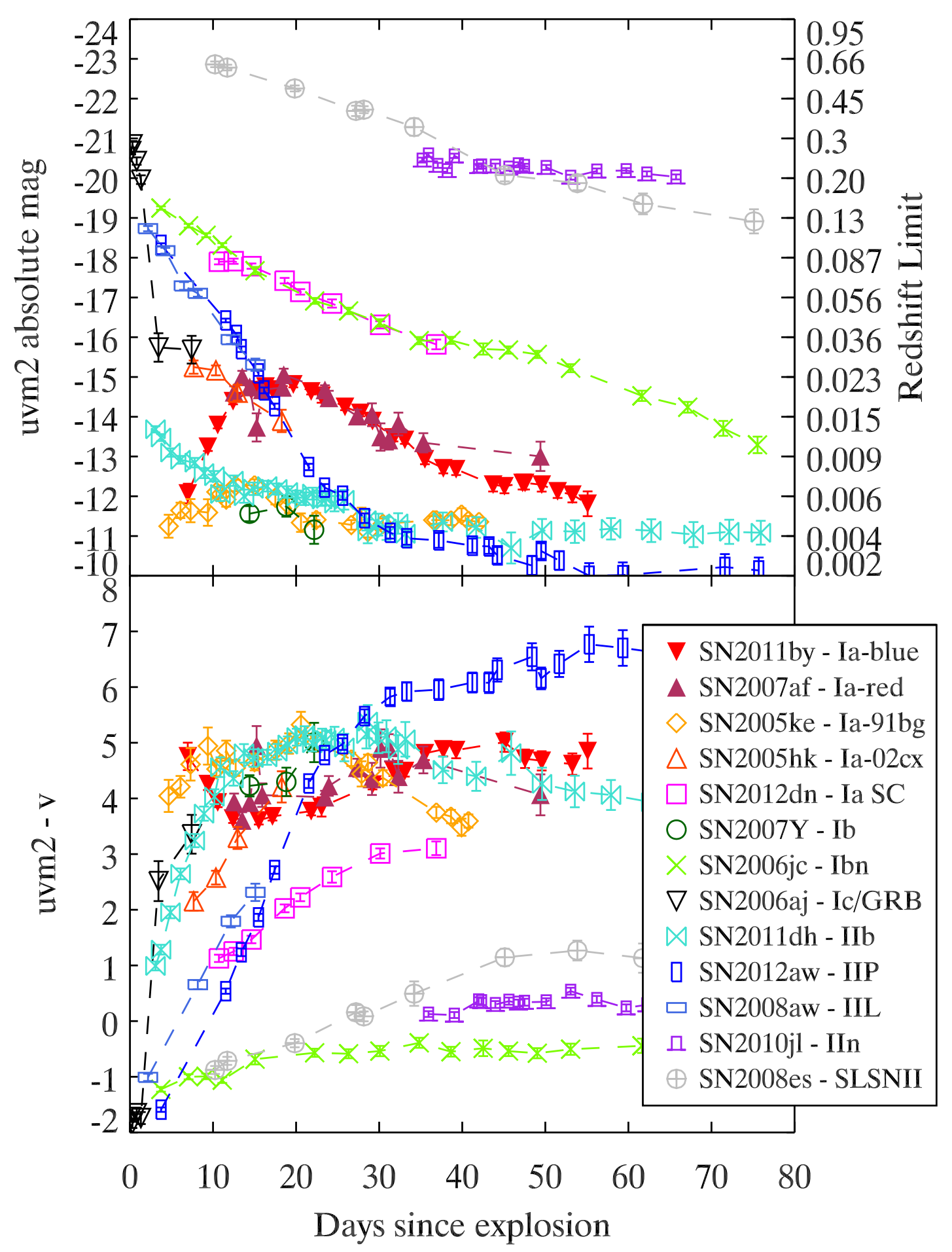

Figure 1: UV absolute magnitudes and UV-optical colors are plotted for prototypes of most supernova types to understand the detectability of supernovae based on the type and age and/or optical magnitude. The redshift limits are based on a uvm2 limiting Vega magnitude of 20. 
Table 1: Swift Supernova Siblings

\begin{tabular}{|c|c|c|c|c|c|c|c|}
\hline Host Galaxy & Redshift & Supernova & Type & Supernova & Type & Supernova & Type \\
\hline M51 & 0.002 & SN2005cs & IIP & SN2011dh & $\mathrm{IIb}$ & $\ldots$ & \\
\hline NGC1316 & 0.006 & SN2006dd & Ia & $\mathrm{SN} 2006 \mathrm{mr}$ & Ia & $\ldots$ & . . \\
\hline NGC7364 & 0.016 & SN2006lc & $\mathrm{Ib} / \mathrm{c}$ & SN2011im & Ia & $\ldots$ & . . \\
\hline MCG+05-43-16 & 0.017 & SN2007ck & IIP & SN2007co & Ia & $\ldots$ & . . \\
\hline NGC4039 & 0.005 & SN2007sr & Ia & SN2013dk & Ic & $\ldots$ & . . \\
\hline NGC2770 & 0.006 & SN2007uy & $\mathrm{Ib}$ & SN2008D & $\mathrm{Ib}$ & $\ldots$ & . \\
\hline ESO121-26 & 0.008 & SN2008M & IIP & SN2009mg & $\mathrm{IIb}$ & SN2012hr & Ia \\
\hline NGC2765 & 0.013 & SN2008hv & Ia & ASASSN-13dd & Ia & $\ldots$ & \\
\hline M61 & 0.005 & SN2008in & IIP & SN2014dt & Ia- $02 c x$ & $\ldots$ & . . \\
\hline NGC1954 & 0.010 & SN2010ko & Ia & SN2011fi & II & SN2013ex & Ia \\
\hline Arp299 & 0.010 & SN2010O & $\mathrm{Ib}$ & SN2010P & $\mathrm{IIb}$ & $\ldots$ & . \\
\hline NGC1566 & 0.005 & SN2010el & Ia- $02 c x$ & ASASSN-14ha & II & $\ldots$ & $\ldots$ \\
\hline NGC6240 & 0.024 & SN2010gp & Ia & PS1-14xw & Ia & $\ldots$ & $\ldots$ \\
\hline NGC2207 & 0.009 & SN2010jp & IIn & SN2013ai & IIP & $\ldots$ & $\ldots$ \\
\hline NGC5806 & 0.005 & SN2012P & Ibc & iPTF13bvn & Ic & $\ldots$ & $\ldots$ \\
\hline NGC7331 & 0.003 & SN2013bu & II & SN2014C & $\mathrm{Ib}$ & $\ldots$ & $\ldots$ \\
\hline
\end{tabular}

Follow up observations of a supernova often result in a higher cadence of galaxy observations, so sometimes an additional supernova in the galaxy is discovered at a younger age than otherwise would have happened. Observations of SN 1999ee in IC5179 led to the very early discovery of SN 1999ex and the first observations of the fading shock breakout of a Ib/c supernova [13]. The best example is the discovery in Swift observations of SN 2007uy of the X-ray shock breakout of SN 2008D [14].

Supernova siblings are important objects in the study of supernova rates as a function of supernova type and host galaxy morphology [15]. They are also important tests of the utility of global (rather than local) environment measurements and their correlation with rates or supernova properties. Sibling supernovae provide independent and joint constraints on the distance to the host galaxy [16] and a direct comparison of the absolute brightness of the siblings without the uncertainties on the distance. In Table 1 we list the 16 host galaxies and the 34 supernovae they have hosted that were observed by Swift. Other multiples have occurred during this period of time, but we are only listing those supernovae observed by Swift.

\section{References}

[1] N. Gehrels, G. Chincarini, P. Giommi, K. O. Mason, J. A. Nousek, A. A. Wells, N. E. White, S. D. Barthelmy, D. N. Burrows, L. R. Cominsky, K. C. Hurley, F. E. Marshall, P. Mészáros, P. W. A. Roming, L. Angelini, L. M. Barbier, T. Belloni, S. Campana, P. A. Caraveo, M. M. Chester, O. Citterio, T. L. Cline, M. S. Cropper, J. R. Cummings, A. J. Dean, E. D. Feigelson, E. E. Fenimore, D. A. Frail, A. S. Fruchter, G. P. Garmire, K. Gendreau, G. Ghisellini, J. Greiner, J. E. Hill, S. D. Hunsberger, H. A. Krimm, S. R. Kulkarni, 
P. Kumar, F. Lebrun, N. M. Lloyd-Ronning, C. B. Markwardt, B. J. Mattson, R. F. Mushotzky, J. P. Norris, J. Osborne, B. Paczynski, D. M. Palmer, H.-S. Park, A. M. Parsons, J. Paul, M. J. Rees, C. S. Reynolds, J. E. Rhoads, T. P. Sasseen, B. E. Schaefer, A. T. Short, A. P. Smale, I. A. Smith, L. Stella, G. Tagliaferri, T. Takahashi, M. Tashiro, L. K. Townsley, J. Tueller, M. J. L. Turner, M. Vietri, W. Voges, M. J. Ward, R. Willingale, F. M. Zerbi, and W. W. Zhang, The Swift Gamma-Ray Burst Mission, ApJ 611 (Aug., 2004) 1005-1020, [astro-ph/].

[2] P. W. A. Roming, T. E. Kennedy, K. O. Mason, J. A. Nousek, L. Ahr, R. E. Bingham, P. S. Broos, M. J. Carter, B. K. Hancock, H. E. Huckle, S. D. Hunsberger, H. Kawakami, R. Killough, T. S. Koch, M. K. McLelland, K. Smith, P. J. Smith, J. C. Soto, P. T. Boyd, A. A. Breeveld, S. T. Holland, M. Ivanushkina, M. S. Pryzby, M. D. Still, and J. Stock, The Swift Ultra-Violet/Optical Telescope, Space Science Reviews 120 (Oct., 2005) 95-142, [astro-ph/].

[3] P. J. Brown, A. A. Breeveld, S. Holland, P. Kuin, and T. Pritchard, SOUSA: the Swift Optical/Ultraviolet Supernova Archive, A\&SS 354 (Nov., 2014) 89-96, [arXiv: 1407.3808$]$.

[4] T. S. Poole, A. A. Breeveld, M. J. Page, W. Landsman, S. T. Holland, P. Roming, N. P. M. Kuin, P. J. Brown, C. Gronwall, S. Hunsberger, S. Koch, K. O. Mason, P. Schady, D. vanden Berk, A. J. Blustin, P. Boyd, P. Broos, M. Carter, M. M. Chester, A. Cucchiara, B. Hancock, H. Huckle, S. Immler, M. Ivanushkina, T. Kennedy, F. Marshall, A. Morgan, S. B. Pandey, M. de Pasquale, P. J. Smith, and M. Still, Photometric calibration of the Swift ultraviolet/optical telescope, MNRAS 383 (Jan., 2008) 627-645, [0 708 . 2259].

[5] A. A. Breeveld, W. Landsman, S. T. Holland, P. Roming, N. P. M. Kuin, and M. J. Page, An Updated Ultraviolet Calibration for the Swift/UVOT, in GAMMA RAY BURSTS 2010. AIP Conference Proceedings (J. E. McEnery, J. L. Racusin, and N. Gehrels, eds.), vol. 1358 of American Institute of Physics Conference Series, pp. 373-376, Aug., 2011. arXiv:1102.4717.

[6] P. J. Brown, P. Kuin, R. Scalzo, M. T. Smitka, M. de Pasquale, S. Holland, K. Krisciunas, P. Milne, and L. Wang, Ultraviolet Observations of Super-Chandrasekhar Mass Type Ia Supernova Candidates with Swift UVOT, ApJ 787 (May, 2014) 29, [arXiv: 1404 . 0650].

[7] P. J. Brown, The Ultraviolet Brightest Type Ia Supernova 2011 de, ApJL 796 (Nov., 2014) L18, [arXiv:1408.1033].

[8] P. J. Brown, P. W. A. Roming, P. Milne, F. Bufano, R. Ciardullo, N. Elias-Rosa, A. V. Filippenko, R. J. Foley, N. Gehrels, C. Gronwall, M. Hicken, S. T. Holland, E. A. Hoversten, S. Immler, R. P. Kirshner, W. Li, P. Mazzali, M. M. Phillips, T. Pritchard, M. Still, M. Turatto, and D. Vanden Berk, The Absolute Magnitudes of Type Ia Supernovae in the Ultraviolet, ApJ 721 (Oct., 2010) 1608-1626, [arXiv: 1007. 4842]. 
[9] K. Krisciunas, P. M. Garnavich, V. Stanishev, N. B. Suntzeff, J. L. Prieto, J. Espinoza, D. Gonzalez, M. E. Salvo, N. Elias de la Rosa, S. J. Smartt, J. R. Maund, and R.-P. Kudritzki, The Type Ia Supernova 2004S, a Clone of SN 2001el, and the Optimal Photometric Bands for Extinction Estimation, AJ 133 (Jan., 2007) 58-72, [astro-ph/].

[10] K. Krisciunas, G. H. Marion, N. B. Suntzeff, G. Blanc, F. Bufano, P. Candia, R. Cartier, N. Elias-Rosa, J. Espinoza, D. Gonzalez, L. Gonzalez, S. Gonzalez, S. D. Gooding, M. Hamuy, E. A. Knox, P. A. Milne, N. Morrell, M. M. Phillips, M. Stritzinger, and J. Thomas-Osip, The Fast Declining Type Ia Supernova 2003gs, and Evidence for a Significant Dispersion in Near-Infrared Absolute Magnitudes of Fast Decliners at Maximum Light, AJ 138 (Dec., 2009) 1584-1596, [arXiv: 0908 .1918].

[11] R. J. Foley and R. P. Kirshner, Metallicity Differences in Type Ia Supernova Progenitors Inferred from Ultraviolet Spectra, ApJL 769 (May, 2013) L1, [arXiv: 1302 . 4479 ].

[12] P. J. Brown, E. Baron, P. Milne, P. W. A. Roming, and L. Wang, Theoretical Clues to the Ultraviolet Diversity of Type Ia Supernovae, ArXiv e-prints (Apr., 2015) [arXiv:1504.0523].

[13] M. Stritzinger, M. Hamuy, N. B. Suntzeff, R. C. Smith, M. M. Phillips, J. Maza, L.-G. Strolger, R. Antezana, L. González, M. Wischnjewsky, P. Candia, J. Espinoza, D. González, C. Stubbs, A. C. Becker, E. P. Rubenstein, and G. Galaz, Optical Photometry of the Type Ia Supernova 1999ee and the Type Ib/c Supernova 1999ex in IC 5179, AJ 124 (Oct., 2002) 2100-2117, [astro-ph/].

[14] A. M. Soderberg, E. Berger, K. L. Page, P. Schady, J. Parrent, D. Pooley, X.-Y. Wang, E. O. Ofek, A. Cucchiara, A. Rau, E. Waxman, J. D. Simon, D. C.-J. Bock, P. A. Milne, M. J. Page, J. C. Barentine, S. D. Barthelmy, A. P. Beardmore, M. F. Bietenholz, P. Brown, A. Burrows, D. N. Burrows, G. Byrngelson, S. B. Cenko, P. Chandra, J. R. Cummings, D. B. Fox, A. Gal-Yam, N. Gehrels, S. Immler, M. Kasliwal, A. K. H. Kong, H. A. Krimm, S. R. Kulkarni, T. J. Maccarone, P. Mészáros, E. Nakar, P. T. O’Brien, R. A. Overzier, M. de Pasquale, J. Racusin, N. Rea, and D. G. York, An extremely luminous X-ray outburst at the birth of a supernova, Nature 453 (May, 2008) 469-474, [0802 . 1712].

[15] J. P. Anderson and M. Soto, On the multiplicity of supernovae within host galaxies, A\&A 550 (Feb., 2013) A69, [arXiv:1212.4153].

[16] M. Stritzinger, C. R. Burns, M. M. Phillips, G. Folatelli, K. Krisciunas, S. Kattner, S. E. Persson, L. Boldt, A. Campillay, C. Contreras, W. Krzeminski, N. Morrell, F. Salgado, W. L. Freedman, M. Hamuy, B. F. Madore, M. Roth, and N. B. Suntzeff, The Distance to NGC 1316 (Fornax A) from Observations of Four Type Ia Supernovae, AJ 140 (Dec., 2010) 2036-2051, [arXiv:1009.4390]. 